\title{
Small intestinal infarction: a fatal complication of systemic oxalosis
}

\author{
J S Johnson, A K Short, A Hutchison, N R Parrott, I S D Roberts
}

\begin{abstract}
Primary hyperoxaluria is a rare genetic disorder characterised by calcium oxalate nephrolithiasis and nephrocalcinosis leading to renal failure, often with extrarenal oxalate deposition (systemic oxalosis). Although ischaemic complications of crystal deposition in vessel walls are well recognised clinically, these usually take the form of peripheral limb or cutaneous ischaemia. This paper documents the first reported case of fatal intestinal infarction in a 49 year old woman with systemic oxalosis and advocates its consideration in the differential diagnosis of an acute abdomen in such patients.

(f Clin Pathol 2000;53:720-721)
\end{abstract}

Keywords: primary hyperoxaluria; oxalosis; intestinal infarction

\section{Case report}

A 49 year old white woman developed rapidly progressive renal failure, diagnosed two months after a total abdominal hysterectomy and bilateral salpingo-oophorectomy for fibroids, requiring haemodialysis four months later. In her past medical history she had two episodes of renal calculi at the ages of 4 and 24 years, which required surgery but were not investigated further. Before the hysterectomy, renal function was normal and histology of the hysterectomy specimen showed no evidence of crystal deposition. Ultrasound scan excluded urinary tract obstruction. A renal biopsy performed after her stabilisation on haemodialysis showed massive oxalate crystal deposition within tubules, with associated tubular obstruction and acute on chronic tubular injury. A clinicopathological diagnosis of primary hyperoxaluria was made. She was subsquently started on pyridoxine and citrate and continuous ambulatory peritoneal dialysis. However, she was intolerant of both these medications and, with her renal function being so poor, both were stopped.

Three months after the initial presentation she developed severe pain in first the left and then the right foot, with clinical signs of local ischaemia bilaterally. Angiography revealed bilateral calf vessel stenosis with single vessel run off to the feet. She then developed an episode of dialysis related peritonitis, which responded well to removal of the Tenckhoff catheter and antibiotic treatment. At the same time, she developed rest pain in both feet, for which amputation was being considered after the failure of medical management with prostacyclin infusions.
Four weeks after recommencing haemodialysis she developed an acute abdomen and an urgent laparotomy was performed, which showed extensive infarction of the small intestine with three separate perforations. Despite resection of the affected bowel and intensive care, she died two days later. A postmortem examination was not performed.

\section{Pathological examination}

The resection specimen comprised $102 \mathrm{~cm}$ of small intestine, with several areas of full thickness perforation on the antimesenteric border, and a fibrinopurulent serosal exudate. The mucosa showed multiple small ulcers and in areas the bowel wall appeared haemorrhagic and thinned. Microscopy revealed widespread mucosal ulceration and acute inflammation with crypt loss, in keeping with ischaemic injury. In addition, there were multiple areas of full thickness infarction. Stains for bacteria and fungi were negative. Under polarised light, numerous birefringent oxalate crystals, with their characteristic radial arrangement, could be seen within the media of the mesenteric arteries and in some smaller submucosal vessels causing lumenal occlusion (fig 1). There was no evidence of thrombosis, embolism, or vasculitis.

\section{Discussion}

Primary hyperoxaluria is a rare autosomal recessive disorder, caused by a functional defect of the liver specific peroxisomal alanine glyoxylate aminotransferase (AGT). The consequent decreased transamination of glyoxylate to glycine leads to a subsequent increase in its oxidation to oxalate, which is a poorly soluble end product. The disorder is characterised by recurrent calcium oxalate nephrolithiasis and nephrocalcinosis, frequently leading to renal failure and death before the age of 20 years. Although more common in early childhood, the age at onset of symptoms ranges from birth to the 6 th decade. ${ }^{1}$ The disease is classified into two types, type I (glycolic aciduria) and type II (L glyceric aciduria), on the basis of the different patterns of urinary organic acid secretion.

Calcium oxalate concentrations are raised in many patients on dialysis, but it is usually only in those patients with primary hyperoxaluria that deposition occurs in extrarenal tissues, despite the use of crystal inhibitors. Postmortem studies have revealed the extent of extrarenal calcium oxalate deposits in this condition, with involvement of bone marrow, cartilage, and myocardium being most common. Crystal deposits have also been described in the prostate gland, testis, thyroid gland, spleen,
Accepted for publication 20 January 2000 

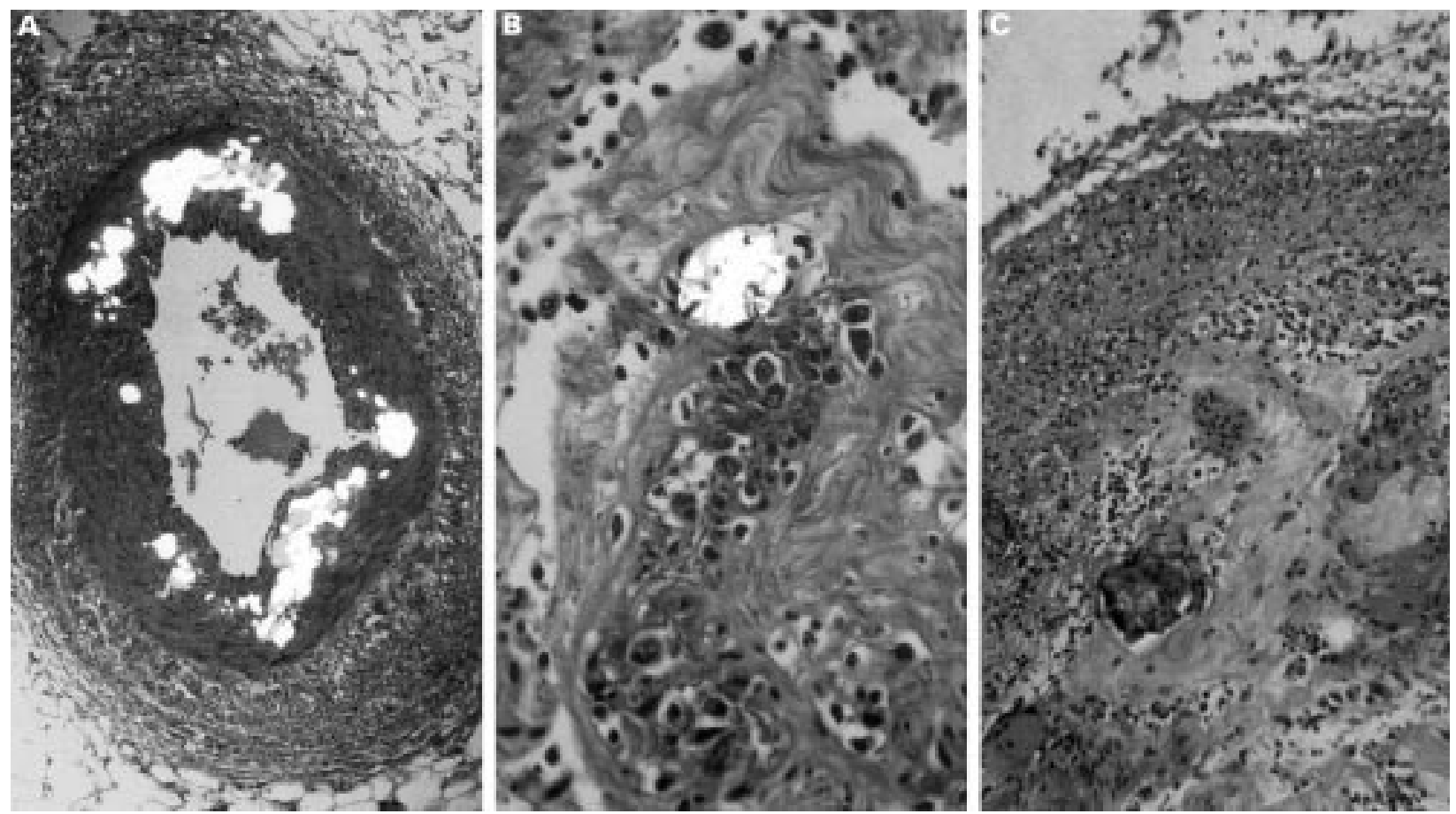

Figure 1 Deposits of oxalate crystals (A) within the media of a mesenteric artery and $(B)$ in a submucosal arteriole. (C) There is mucosal necrosis and ulceration, associated with oxolate crystal deposition within the submucosa. Haematoxylin and eosin stained;polarised light.

liver, thymus, pituitary gland, adrenal gland, pancreas, and the central and peripheral nervous systems. ${ }^{23}$ In most of these cases, the deposits were limited to the vessel wall, with arterial and arteriolar involvement being more common than venous involvement.

Reported cases of ischaemic damage secondary to vessel wall involvement include the sudden onset of peripheral ischaemia with livedo reticularis, necessitating amputation of a metatarsal, in a 38 year old $\operatorname{man}^{4}$ and cutaneous gangrene..$^{5}$ This is the first reported case of intestinal infarction secondary to systemic oxalosis. Other possible causes of infarction include mesenteric thrombosis, atheroembolism, and vasculitis. An unusual cause of intestinal infarction in patients with renal failure is necrosis associated with Kayexalate in sorbitol, administered orally in the treatment of hyperkalaemia. ${ }^{6}$ In this case, there was good flow within the main mesenteric arteries at the time of laparotomy, and histology of the resected specimen excluded other vascular lesions. Therefore, we conclude that infarction was a direct consequence of microvascular occlusion associated with massive oxalate crystal deposition.

The presumed mechanism of the ischaemia is endothelial damage with subsequent spasm after in situ crystallisation as a result of saturating blood concentrations of calcium and oxalate. Contact of plasma with the crystal surface leads to activation of serum complement, with subsequent triggering of neutrophil mediated endothelial cell damage as a result of the release of toxic oxygen radicals. Platelets are thought to augment the endothelial damage by release of their products, with serotonin being particularly implicated.

In our patient, it is possible that her calcium oxalate concentrations in the past had rarely exceeded those required for precipitation, and that it was only the metabolic stresses of a surgical procedure that led to her rapid renal insufficiency. The various treatment strategies are discussed comprehensively elsewhere, ${ }^{1}$ but the main aim is early diagnosis and treatment with crystal inhibitors and diuresis to prevent further renal damage. Combined liver-kidney transplantation has produced good results in several patients, with some data suggesting a decline in arterial calcium oxalate deposition post-transplantation. Unfortunately, in our patient the development of systemic complications precluded any assessment for transplantation.

1 Cochat P. Nephrology forum: primary hyperoxaluria type I. Kidney Int 1999;55:2533-47.

2 Williams HE, Smith LH. Primary hyperoxaluria. In: Stanbury JB, Wyngaarden, JB, Fredrickson DS, et al, eds. The metabolic basis of inherited disease, 5 th ed. New York: McGraw-Hill, 1983.

3 Baethge BA, Sanusi ID, Landreneau MD, et al. Livedo reticularis and peripheral gangrene associated with primary hyperoxaluria. Arthritis Rheum 1988;31:1199-203.

4 Boquist L, Lindqvist B, Ostberg Y, et al. Primary oxalosis. Am $\mathcal{F}$ Med 1973;54:673-81.

5 Somachs SC, Davis BR, Paras FA, et al. Fatal cutaneous necrosis mimicking calciphylaxis in a patient with type I primary hyperoxaluria. Arch Dermatol 1995;131:821-3.

6 Rashid A, Hamilton SR. Necrosis of the gastrointestinal tract in uremic patients as a result of sodium polystyrene sulfonate (Kayexalate) in sorbitol: an underrecognised condition. Am F Surg Pathol 1997;21:60-9.

7 Boogaerts MA, Hammerschmidt DE, Roelant C, et al. Mechanisms of vascular damage in gout and oxalosis: crystal induced, granulocyte-mediated endothelial injury. Thromb Haemost 1983;50:576-80. 